\title{
Health care provider preferences for, and barriers to, cannabis use in cancer care
}

\author{
A. McLennan BA(Hons), ${ }^{*}$ M. Kerba MD MPA, ${ }^{+}$U. Subnis $\mathrm{PhD}^{*}$ T. Campbell $\mathrm{PhD}^{*}$ and L.E. Carlson $\mathrm{PhD}^{*}$
}

\begin{abstract}
Background Limited research has been conducted about the perspectives of oncology health care providers (HCPs) concerning the use of cannabis in cancer care and their potential role in advising patients. We sought to determine the barriers encountered by HCPs with respect to medical cannabis and their preferred practices in this area.

Methods An anonymous survey about cannabis was distributed to oncology HCPs at the Tom Baker Cancer Centre in Calgary, Alberta. The 45-question survey measured the opinions of HCPs about cannabis use and authorization in oncology.

Results Of 103 oncology HCPs who participated in the study, 75\% were women. By HCP type, the most commonly reported professional groups were oncology nurse (40\%), radiation therapist (9\%), and pharmacist (6\%). Of respondents, $75 \%$ reported providing direct care to cancer patients. More than half $(69 \%)$ had spoken to a patient about cannabis in the preceding month, and $84 \%$ believed that they lacked sufficient knowledge about cannabis to make recommendations. Barriers such as monitoring the patient's use of cannabis (54\%), prescribing an accurate dose $(61 \%)$ or strain $(53 \%)$, and having insufficient research $(50 \%)$ were most commonly reported. More than half of HCPs (53\%) would be interested in receiving more information or training about the use of cannabis in oncology.
\end{abstract}

Conclusions The survey indicated that this group of oncology HCPs believed that they lacked sufficient knowledge about cannabis to make recommendations to patients. In addition to that lack of knowledge, a number of notable barriers were reported, and more than half the HCPs indicated interest in learning more about cannabis in the future.

Key Words Cannabis, knowledge, preferences, barriers

Curr Oncol. 2020 April:27(2)e199-e205

www.current-oncology.com

\section{INTRODUCTION}

Cannabis sativa is a pharmacologically complex plant, containing more than 100 different cannabinoid compounds ${ }^{1}$. Cannabinoids, which are derived from the plant (phytocannabinoids) and found in the body (endocannabinoids), exhibit a wide array of functions and interactions, most of which that have yet to be elucidated ${ }^{1}$. Of the many compounds, THC ( $\Delta-9$-tetrahydrocannabinol), and CBD (cannabidiol) have been widely researched as medicinal agents because they have been discovered to have therapeutic potential ${ }^{2}$.

Recent literature indicates that cannabis use by people living with cancer has become more frequent and that many of those individuals are using it to mitigate and manage symptoms related to their condition and treatments ${ }^{3-5}$. Some of the most commonly reported symptoms are cancer-related pain (including acute, chronic, and neuropathic pain), chemotherapy-induced nausea and vomiting, appetite loss, sleep loss, and emotional symptoms such as anxiety and depression ${ }^{6-9}$.

Although the literature is growing, it is important to emphasize that cannabis-based research is in its infancy and that many research findings should be interpreted cautiously ${ }^{9}$. The uncertainty has contributed to a degree of reasonable concern voiced by oncology health care providers (HCPs) ${ }^{4}$. Furthermore, many oncology HCPs who were trained during the pre-legalization era were not educated about the potential uses of cannabis as a medicine; much of the prior literature viewed cannabis as a drug of abuse, rather than as a potential therapeutic agent ${ }^{5}$. A qualitative study found that the opinions of oncology experts about cannabis are divided between those who support and 
those who discourage its use in oncology $y^{4}$. Another recent study found that only $30 \%$ of oncologists believe that they have sufficient knowledge to make recommendations to patients about cannabis use, although the same study found that $80 \%$ of oncologists had spoken to a patient about cannabis and that $46 \%$ had made at least 1 cannabis-based recommendation to a patient in the month preceding the study ${ }^{10}$.

Other HCPs have indicated that the lack of well-defined effect specifications exposes patients to a wide range of experiences when using medical cannabis, rather than to a well-defined and controlled pharmacologic effect ${ }^{4}$. Very few epidemiology studies have looked at the potential negative health effects of cannabis, and although some proponents suggest that the common side effects are rather benign, recent research has identified various health risks potentially associated with cannabis use, many of which are subject to individual factors, such as the patient's age, physical and mental health condition, and past experiences with substance use, among others ${ }^{4,11}$. Given the rapid pace at which cannabis has been legalized and adopted by various patients, physicians and other HCPs are challenged about how to knowledgably address questions, provide professional recommendations, and confidently authorize medical cannabis ${ }^{10}$.

Hence, the current situation for Canadian cancer patients and oncology HCPs is that patients are interested in the use of medical cannabis for treating symptoms of pain, nausea and vomiting, and so on; that many are already using it; and that access has become much easier with the legalization of recreational cannabis, but that patients are largely unguided and are using cannabis without authorization or recommendation from their oncology health care team. Oncology HCPs have identified a number of barriers with respect to the use of cannabis by their patients, such as an unpredictable range of effects, a lack of understanding about its health benefits and risks, and an absence of professional knowledge about its use overall.

The aim of the present study was therefore to identify approaches that facilitate the appropriate authorization and use of medical cannabis in cancer care. Our primary objective was to investigate knowledge, beliefs, barriers, and preferences on the part of oncology HCPs with respect to use of medical cannabis by their patients.

\section{METHODS}

\section{Survey Design}

An anonymous survey was designed to elicit information from oncology HCPs about use of cannabis by their patients. The survey included questions relating to professional knowledge about cannabis, clinical skills and preparation, information-seeking, professional beliefs, clinical barriers faced, and preferences related to the provision of cannabis-based education and professional training opportunities.

The survey consisted of 45 questions pertaining to 6 areas of interest: knowledge, clinical skills, and preparation; information-seeking; beliefs and practices; barriers faced; preferences; and demographics. Certain questions-all those in section 1 (knowledge and skills), both in section 2 (information-seeking), all in section 3 (beliefs and practices), and both in section 5 (preferences)-were taken from a survey concerning complementary and alternative medicines and treatments ${ }^{12}$. All other questions were created by the research team.

\section{Participants}

Eligible participants were HCPs employed by Alberta Health Services, Cancer Control Alberta, working with cancer patients either directly or indirectly.

Participants were approached at the Tom Baker Cancer Centre in Calgary and were notified about the study by Alberta Health Services e-mail to all Cancer Control Alberta staff in the Calgary area. They were asked to complete the online HCP cannabis survey and were provided with a link. E-mail messages were sent to all oncology staff members at Tom Baker Cancer Centre-approximately 300 HCPs-to attain a representative sample of the target population. Reminders were sent by e-mail to potential participants before and throughout the data collection period. The survey was anonymous, and participants were not asked to disclose their name or any other identifiable information. Distribution of invitations and collection of data began in December 2018, with subsequent e-mail reminders sent out in January and February 2019.

\section{Statistical Methods}

Data were summarized using descriptive statistics (means with standard deviations for continuous variables, and percentages and ranges for categorical variables). Subgroups (men vs. women, knowledgeable vs. unknowledgeable, and younger vs. older practitioner age) were compared using independent-samples $t$-tests or analysis of variance for continuous variables and chi-square tests for categorical variables (where responses allowed) to look for differences in outcomes (knowledge, beliefs, barriers, and preferences) between subgroups. A $p$ value less than or equal to 0.05 was regarded as statistically significant.

\section{Ethics Considerations}

Before the surveys were distributed, the project was reviewed and approved by the Health Research Ethics Board of Alberta (Cancer Care ID 18-0440).

\section{RESULTS}

\section{Response Rate}

From a population sample of approximately $300 \mathrm{HCPs}$ at the Tom Baker Cancer Centre, all of whom received an e-mail invitation to the survey, 103 completed the survey, for a response rate of $0.34(34 \%)$.

\section{HCP Demographics}

Table I presents demographic characteristics for the 103 HCPs who completed the online questionnaire. Of those who responded, 15 (15\%) identified as male, and 77 (75\%), as female; $11(11 \%)$ left the gender identification section blank. Most HCPs indicated that they were white $(n=73,71 \%)$ and that Canada was their country of birth $(n=79,77 \%)$ For HCP type, $75 \%$ of the respondents $(n=77)$ indicated that they provide direct care to cancer patients. The most commonly 
reported disciplines were oncology nurse $(n=42,41 \%)$, radiation therapist $(n=9,9 \%)$, and pharmacist $(n=6,6 \%)$.

\section{HCP Knowledge}

Participants were asked to indicate their degree of knowledge about medical cannabis with respect to its use in cancer care. For the role of medical cannabis in symptom management, 24 participants $(23 \%)$ reported being "knowledgeable," and 65 (63\%) "somewhat knowledgeable.” For knowledge of empirical evidence about cannabis, 14 (14\%) reported being "knowledgeable"; 48 (47\%), "somewhat knowledgeable"; and 40 (39\%), "not at all knowledgeable." The HCPs were also asked if they believed they had sufficient knowledge about medical cannabis to make recommendations to patients. It was the belief of 85 HCPs (83\%) that they lacked sufficient knowledge to do so (Table II).

A significant relationship was found between HCP knowledge and age, as determined by analysis of variance, $F_{(6,98)}=145.37, p=0.005$. A Tukey honest significant difference test revealed that, compared with HCP participants

TABLE I Demographic characteristics of responding health care providers (HCPs)

\begin{tabular}{|c|c|c|}
\hline \multirow[t]{2}{*}{ Characteristic } & \multicolumn{2}{|c|}{ Value } \\
\hline & (n) & $(\%)$ \\
\hline \multicolumn{3}{|l|}{ Sex } \\
\hline Male & 15 & (14.6) \\
\hline Female & 77 & $(74.8)$ \\
\hline Other & 0 & $(0.0)$ \\
\hline Missing & 11 & $(10.7)$ \\
\hline \multicolumn{3}{|l|}{ Age group } \\
\hline 18-29 Years & 16 & (15.5) \\
\hline 30-39 Years & 28 & $(27.2)$ \\
\hline 40-49 Years & 21 & (20.4) \\
\hline 50-59 Years & 18 & $(17.5)$ \\
\hline 60-69 Years & 7 & $(6.7)$ \\
\hline $70-79$ & 2 & (1.9) \\
\hline Missing & 11 & $(10.7)$ \\
\hline \multicolumn{3}{|l|}{ HCP type } \\
\hline Medical oncologist & 5 & $(4.9)$ \\
\hline Radiation oncologist & 5 & $(4.9)$ \\
\hline Gynecological oncologist & 1 & $(1.0)$ \\
\hline Nurse & 42 & $(40.8)$ \\
\hline Palliative care practitioner & 5 & $(4.9)$ \\
\hline Administrative & 7 & $(6.8)$ \\
\hline Radiation therapist & 9 & $(8.7)$ \\
\hline Pharmacist & 6 & $(5.8)$ \\
\hline Social worker & 1 & $(0.9)$ \\
\hline Dietitian & 1 & $(0.9)$ \\
\hline Rehabilitation oncology & 2 & (1.9) \\
\hline Research & 2 & $(1.9)$ \\
\hline Psychologist & 3 & $(2.9)$ \\
\hline Missing & 14 & (13.6) \\
\hline \multicolumn{3}{|l|}{ Direct care to patients } \\
\hline Yes & 77 & (74.8) \\
\hline No & 16 & (15.5) \\
\hline Missing & 10 & (9.7) \\
\hline
\end{tabular}

$30-39$ years of age $(11.39 \pm 3.06)$, those $50-59$ years of age $(8.72 \pm 2.54)$ reported being significantly less knowledgeable about medical cannabis. Another statistically significant relationship was observed between HCP knowledge and HCP type: $\chi^{2}{ }_{(6)}=13.865, p=0.031$. Of the oncology nurse respondents, $90 \%$ reported having insufficient knowledge about medical cannabis to make recommendations to patients.

\section{Clinical Preparedness}

Participants were asked to indicate their level of preparedness from 1, "not at all," to 4 , "very" (7.28 \pm 3.01$)$. When asked how prepared they felt to monitor use of cannabis by their patients, 71 (69\%) reported feeling "not at all prepared," and when asked about assisting patients in making decisions about cannabis, 62 (60\%) reported feeling "not at all prepared." Regarding preparedness to critique cannabis-based academic sources, respondents 50-59 years of age indicated being significantly less prepared than did respondents $30-39$ years of age: $\chi_{(18)}^{2}=30.46, p=0.033$.

\section{HCP Beliefs and Practices}

Table III presents data about HCP practices. The HCPs were questioned about patient interactions during the month preceding taking the questionnaire, with 71 (69\%) indicating that a patient had told them about their use of cannabis, and $42(41 \%)$ indicating that a patient had asked for their

TABLE II Health care provider knowledge about cannabis

\begin{tabular}{lll}
\hline \multicolumn{1}{c}{ Question } & \multicolumn{2}{c}{ Response } \\
\cline { 2 - 3 } & $(\boldsymbol{n})$ & $\mathbf{( \% )}$ \\
\hline $\begin{array}{l}\text { Do you believe that you have sufficient } \\
\text { knowledge about cannabis to make } \\
\text { recommendations to patients? }\end{array}$ & & \\
Yes & 17 & 16.5 \\
No & 86 & 83.5 \\
\hline
\end{tabular}

TABLE III Health care provider practices with respect to cannabis

\begin{tabular}{lcc}
\hline \multicolumn{1}{c}{ Question } & \multicolumn{2}{c}{ Yes response $^{\mathrm{a}}$} \\
\cline { 2 - 4 } & $(\boldsymbol{n})$ & $\mathbf{( \% )}$ \\
\hline In the past month, did you ... & & \\
\hline Ask a patient about their use of cannabis? & 49 & 51.5 \\
\hline $\begin{array}{l}\text { Provide educational material to cancer patients } \\
\text { about cannabis? }\end{array}$ & 6 & 5.8 \\
$\begin{array}{l}\text { Review evidence about cannabis with a patient? } \\
\begin{array}{l}\text { Suggest that a patient use cannabis as part of } \\
\text { their treatment? }\end{array}\end{array}$ & 9 & 19.4 \\
\hline
\end{tabular}

In the past month, did a patient ...

\begin{tabular}{lccc}
\hline Tell you about their use of cannabis? & 71 & 68.9 \\
\hline Ask you for your advice about cannabis? & 42 & 41.2 \\
\hline Ask you to monitor their use of cannabis? & 5 & 4.9 \\
\hline Ask you to refer them to a cannabis practitioner? & 28 & 27.5 \\
\hline a Questions solicited "yes" or "no" answers. & &
\end{tabular}


advice about cannabis use. The HCPs were asked if, in the past month, they had referred a patient to another HCP for further information about cannabis, and 61 (59\%) reported that they had made such a referral. The most commonly reported "referred to" HCPs were an oncologist ( $n=36$, $35 \%)$, a medical cannabis practitioner $(n=34,33 \%)$, and a pharmacist ( $n=17,17 \%)$.

Participants were asked to indicate their level of agreement with statements related to cannabis. Agreement that cannabis is a useful supplement to some conventional medications was indicated by 40 respondents (39\%), and agreement that cannabis could be used in addition to existing cancer treatments, by 49 (48\%). Respondents were also asked to indicate how effective it would be to include cannabis in a patient's treatment regimen (in comparison with conventional treatments) for various cancer-related symptoms. For nausea and vomiting, 14 (14\%) indicated "much more effective"; 43 (42\%), "somewhat effective"; and 5 (5\%), "much less effective." For cancer-related pain, 12 (12\%) indicated "much more effective"; 43 (42\%), "somewhat effective"; and 14 (14\%), "much less effective." And for sleep, 20 (19\%) indicated "much more effective"; 38 (37\%) "somewhat effective"; and 5 (5\%), "much less effective." Table IV presents further data.

A considerable number of HPCs-24 (23\%) — had asked a patient to stop using cannabis in the preceding month. Those respondents commonly reported reasons such as evidence or literature about interaction or harm ( $n=9$, $9 \%$ ), theoretical concerns about interactions with other medications ( $n=9,9 \%)$, and no evidence of efficacy $(n=5$, $5 \%$ ). Risk or evidence of negative side effects and harm to lungs were also specified as additional reasons.

\section{HCP Barriers}

Participating HCPs were provided a list of choices and were asked to indicate those that they believed to be barriers to medical cannabis use in health care. The barriers most commonly reported were difficulties prescribing the proper doses ( $n=63,61 \%$ ), difficulties actively monitoring a patient's use of cannabis ( $n=56,54 \%)$, difficulties prescribing the proper strains ( $n=55,53 \%)$, and negative conventional beliefs toward cannabis ( $n=55,53 \%)$. Additionally, $52(50 \%)$ indicated that a lack of significant or credible research was a barrier, and 49 (48\%) reported that a lack of support from the medical community was a barrier. Table $\mathrm{v}$ presents further data.

\section{Preferences}

Frequency calculations showed that 82 HCPs $(80 \%)$ would be "very interested" or "somewhat interested" in receiving more information, education, or training about medical cannabis. Only 15 (15\%) indicated they would be uninterested. When asked about the types of services they would prefer, 25 (24\%) indicated information seminars about the effectiveness and safety of medical cannabis; 20 (19\%), online webinars; 21 (20\%), training programs about medical cannabis use in cancer care; and 13 (13\%), an information booklet about the effectiveness and safety of medical cannabis (Table VI). Online self-learning modules, patient testimonials, and systematic reviews were also specified by respondents.

\section{DISCUSSION}

Our study found that, overall, HCPs at Alberta Health Services, Cancer Control Alberta, in Calgary were only moderately knowledgeable about cannabis and its utility in cancer. Our study is unique in that it shows that, of oncology HCPs, almost all oncology nurses have insufficient knowledge. However, despite that lack of knowledge, far more than half the participants reported that they had spoken to a patient about cannabis in the month preceding this study, and far more than half had provided recommendations to their patients. Compared with their younger colleagues, older HCPs self-reported less knowledge about cannabis and less preparation to critique cannabis-based literature. With respect to treatment efficacy, the general belief was that it could be used effectively as an adjunct with existing medications, and many respondents believed that it is effective in treating symptoms of chemotherapy-induced nausea and vomiting, pain, and sleep problems. Commonly reported barriers included concerns about monitoring how patients use cannabis, about prescribing accurate doses or proper strains, and about the lack of existing credible research and overall support from the medical community. Further, we found that a large proportion of HCPs are interested in receiving more professional training and education about the uses of cannabis in oncology.

TABLE IV Health care provider opinions about cannabis as a treatment for cancer-related symptoms

\begin{tabular}{|c|c|c|c|c|c|}
\hline \multirow[t]{2}{*}{ Symptom } & \multicolumn{5}{|c|}{$\begin{array}{l}\text { Compared with standard practice, implementing cannabis in a patient's } \\
\text { treatment regimen to treat this cancer-related symptom is ... } \\
{[n(\%)]}\end{array}$} \\
\hline & $\begin{array}{c}\text { Much } \\
\text { more effective }\end{array}$ & $\begin{array}{c}\text { Somewhat } \\
\text { more effective }\end{array}$ & $\begin{array}{l}\text { Equally } \\
\text { effective }\end{array}$ & $\begin{array}{l}\text { Somewhat } \\
\text { less effective }\end{array}$ & $\begin{array}{c}\text { Much } \\
\text { less effective }\end{array}$ \\
\hline Depression & $6(5.8)$ & $26(25.2)$ & $9(8.7)$ & $9(8.7)$ & 16 (15.5) \\
\hline Sleep & $20(21.4)$ & $38(36.9)$ & $7(6.8)$ & $11(10.7)$ & $5(4.9)$ \\
\hline Nausea or vomiting & $14(13.6)$ & $43(41.7)$ & $9(8.7))$ & $11(10.7)$ & $5(4.9)$ \\
\hline Weight gain & $5(4.9)$ & $26(25.2)$ & $12(11.7)$ & 14 (13.6) & $7(6.8)$ \\
\hline Anxiety & $14(13.6)$ & $39(37.9)$ & $7(6.8)$ & $8(7.8)$ & $12(11.7)$ \\
\hline Cancer-related pain & $12(11.7)$ & $43(41.7)$ & $6(5.8)$ & $10(9.7)$ & $14(13.6)$ \\
\hline
\end{tabular}




\section{Knowledge}

The survey results demonstrate that oncology HCPs lack sufficient knowledge about cannabis and its utility as a medication in cancer treatment. Our findings are interesting, in that we found that almost all oncology nurses reported having insufficient knowledge to make recommendations to patients, which likely indicates a lack of pharmacology training and education about the uses of cannabis during nursing training in particular, which might also extend to medical training. Braun et al. ${ }^{10}$ similarly found that fewer than half the oncologists in their sample believed that they had sufficient knowledge, although $80 \%$ had conducted discussions with their patients about cannabis and almost half had recommended cannabis use for at least 1 patient in the preceding year.

Abrams ${ }^{5}$ argues that many HCPs trained during the prohibition era are likely to be unaware of the potential uses of cannabis as a medicine, and our findings align with that opinion. Compared with their younger counterparts, older HCPs reported being less knowledgeable and feeling less prepared to critique cannabis-based academic sources-specifically, the 30-39 and 50-59 cohorts, which might

TABLE V Health care provider barriers with respect to cannabis

\begin{tabular}{|c|c|c|}
\hline \multirow[t]{2}{*}{ Barrier } & \multicolumn{2}{|c|}{ Response } \\
\hline & $(n)$ & $(\%)^{\mathrm{a}}$ \\
\hline Lack of efficacy or objective effects & 46 & 44.7 \\
\hline Insignificant research & 52 & 50.5 \\
\hline $\begin{array}{l}\text { Difficulties prescribing proper strains of cannabis } \\
\text { to patients }\end{array}$ & 55 & 53.4 \\
\hline Difficulties prescribing proper doses to patients & 63 & 61.2 \\
\hline $\begin{array}{l}\text { Difficulties in actively monitoring patient's use of } \\
\text { cannabis }\end{array}$ & 56 & 54.4 \\
\hline Lack of support from medical community & 49 & 47.6 \\
\hline Negative conventional beliefs about cannabis & 55 & 53.4 \\
\hline Other & 6 & 5.8 \\
\hline
\end{tabular}

a More than 1 barrier could be selected.

TABLE VI Health care provider preferences with respect to cannabis

\begin{tabular}{lll}
\hline \multicolumn{1}{c}{ Preference } & \multicolumn{2}{c}{ Response } \\
\cline { 2 - 3 } & $(\boldsymbol{n})$ & $\mathbf{( \% )}^{\mathbf{a}}$ \\
\hline $\begin{array}{l}\text { Information seminars about the effectiveness } \\
\text { and safety of cannabis }\end{array}$ & 25 & 24.3 \\
$\begin{array}{l}\text { Online webinars about the effectiveness and } \\
\text { safety of cannabis }\end{array}$ & 20 & 19.4 \\
$\begin{array}{l}\text { An information booklet about the } \\
\text { effectiveness and safety of cannabis }\end{array}$ & 13 & 12.6 \\
$\begin{array}{l}\text { Clinical training programs about the use of } \\
\text { cannabis in cancer care }\end{array}$ & 21 & 20.4 \\
\hline $\begin{array}{l}\text { Easier access to peer-reviewed sources } \\
\text { about cannabis }\end{array}$ & 7 & 6.8 \\
\hline \begin{tabular}{l} 
Other \\
\hline
\end{tabular}
\end{tabular}

a More than 1 preference could be selected. partly be attributable to the period when they received their training as HCPs. In another recent survey study of physicians-in-training and medical faculty deans, Evanoff et al. ${ }^{13}$ found that $67 \%$ of medical school curriculum deans reported that their graduate students were not prepared to prescribe cannabis to patients, and $48 \%$ strongly agreed that information about cannabis should be included in undergraduate-level education for medical students. Such recent reports indicate that cannabis education should be implemented and introduced to HCPs earlier on-perhaps even at the undergraduate level-and should be included in nursing curricula as well as in medical training. Although training and educating practicing oncology physicians and other oncology HCPs is effective and necessary, educating students early on would be an advantageous step for avoiding many of the existing knowledge gaps.

\section{Beliefs and Practices}

Although most of our participants (69\%) indicated that a patient had, in the preceding month, asked them about using cannabis, and two thirds had referred a patient to another HCP to acquire more information, considerably fewer HCPs (fewer than half) had asked a patient about cannabis, which suggests that patients are more likely to initiate such conversations. That finding is not uncommon. A 2014 survey study of oncologist beliefs about herbs and supplemental medicines found that only $41 \%$ of oncologists had spoken with their patients about complementary and alternative medicine, and only $26 \%$ had initiated those conversations ${ }^{14}$. Another study by Ziemianski et al ${ }^{15}$ found that $79 \%$ of HCPs reported being approached by a patient to discuss cannabis, with $39 \%$ of the HCPs initiating the discussion.

The lack of awareness on the part of most HCPs about cannabis use and uncertainty about its use as a medication likely explain why HCPs less frequently initiate conversations about cannabis. Although studies show that more than $85 \%$ of HCPs believe proper communication enhances the patient-practitioner relationship, others indicate that a lack of education and training hinders sufficient communication between HCPs and their patients ${ }^{14,16}$. It has been found that insufficient patient-practitioner communication discourages patients from asking questions, increases stress, and can lead to feelings of helplessness; proper communication promotes trust and facilitates control and internal motivation for patients to manage their own conditions, subsequently resulting in better health outcomes ${ }^{17}$. Those observations are directly relatable to the situation of patients seeking information about cannabis use, because they might be less likely to ask questions and to gain information if they perceive their HCP to be unknowledgeable. If they are referred to another HCP, they could become frustrated, because seeking out and receiving the wanted information can add time and stress to an already-filled schedule. Oncology nurses might find adequate education and training about how to communicate cannabis-based information to patients to be of particular importance, considering that they are routinely the front line for addressing questions or concerns, and they oftentimes maintain a strong degree of trust with their patients ${ }^{18}$.

Although oncology HCPs in the present study lacked sufficient knowledge, many believed that cannabis could 
be effectively used for certain cancer-related symptoms. Braun et al. ${ }^{10}$ found that $67 \%$ of the oncologists they surveyed believed that cannabis is a useful and effective adjunct to existing analgesic medications and that $65 \%$ believed cannabis to be equally effective at enhancing appetite. According to Abrams ${ }^{19}$, although oncologists are cautious about prescribing and recommending botanical products to patients, the results of recent studies are encouraging, and it is likely that further investigation will substantiate those findings.

\section{Clinical Barriers}

Oncology HCPs reported a number of noteworthy barriers that they face when dealing with cannabis in practicespecifically, ability to monitor its use, ability to prescribe accurate doses, negative conventional beliefs, and lack of credible research. Braun et al. ${ }^{4}$ reported that reluctance on the part of oncologists to prescribe cannabis results partly from the anticipated exposure of patients to a broad and unpredictable experience during use, rather than to a well-defined pharmacologic effect, which is typically preferred and expected when prescribing medications. Oncologists are accustomed to seeing strong evidence supporting a therapeutic intervention and are oftentimes reluctant to recommend or prescribe medications that have not been approved through rigorous analysis-even if those medications are federally approved ${ }^{5}$. The HCPs in our study were concerned about cannabis interacting poorly with other cancer medications, about associated respiratory and cardiovascular risks, and about other subjective risks that might be linked to the condition of their patients. $\mathrm{Hall}^{20}$ has suggested that various acute and long-term adverse health effects have been observed in association with cannabis use, indicating that its use might be a risk for select populations; however, the risks he enumerated were specific to recreational users and might not apply to those using it for therapeutic purposes. Braun et al. ${ }^{4}$ infers that elderly patients and those with a history of cognitive impairment or mental illness might be at a higher risk of experiencing adverse effects (dizziness, nausea, psychosis, etc.). Those concerns are cogent and demand the attention of researchers and HCPs, because the use of cannabis might be more of a health risk than a health benefit for some cancer patients. Notwithstanding those concerns, patients oftentimes indicate that the adverse effects they experience from cannabis are benign and easily managed, especially in comparison with those of the other medications and treatments they experience ${ }^{9}$.

\section{Preferences}

There is a need for more robust cannabis-based training and education opportunities in oncology. Fortunately, studies show that most HCPs want to learn more about cannabis ${ }^{10}$. Ziemianski et al. ${ }^{15}$ found that HCPs would most prefer to receive educational information through peer-reviewed literature reviews, online learning programs, and small workshops or programs. In another recent assessment of cannabis knowledge among Canadian oncology nurses, Balneaves et $a l .{ }^{21}$ found that $76.8 \%$ of respondents indicated a strong need for better education about cannabis for therapeutic purposes; further, respondents indicated that they would feel more confident authorizing cannabis for patients if sufficient education was provided to them. These HCPs would most prefer access to online learning programs and resources, together with workshops, newsletters, and expert presentations. Our findings also show that the use of online education programs such as webinars or self-learning modules would be an effective and efficient method to reach a large number of HCPs. Furthermore, the use of in-person training programs could be an effective strategy to provide communication guidelines for HCPs to comfortably talk with their patients about cannabis, considering that one existing barrier is the quality of communication between patients and HCPs.

\section{Limitations}

Some limitations of the present study are that a large proportion of the HCP sample consisted of oncology nurses, leaving other HCPs such as oncologists underrepresented. Although our data still yielded interesting and meaningful results, those results might not be generalizable to all oncology HCPs in Calgary. Furthermore, considering that our survey was anonymous, we had no way to distinguish between respondent and non-respondent characteristics, which might have left the study vulnerable to non-response bias. Survey questions were not vetted beforehand, but most questions were drawn from previously validated surveys. A final limitation was the sole use of electronic media to recruit HCPs, which could have excluded HCPs who do not frequently check e-mail. Notwithstanding the aforementioned limitations, our study did yield interesting and valuable information related to the challenges faced by oncology HCPs to adapt to the prevalence of cannabis in the Alberta cancer care system.

\section{Clinical Implications and Future Considerations}

Cannabis-based research in oncology remains in its infancy. The avenues that researchers could take to better understand this complex plant and its implications in cancer care are therefore numerous. Our study presents important new findings that support the need to provide better information and training about cannabis for oncology HCPs, including how to evaluate cannabis-based literature, the primary uses of cannabis in cancer, and how to effectively communicate that knowledge to patients and to safely authorize cannabis use. A logical next step would be to further investigate the aforementioned barriers experienced by HCPs (prescribing strains and doses of cannabis and monitoring use by the patient) to better understand how those barriers can be addressed and in which ways cannabis should be considered in the future. Focusing on preferences, it would be useful to better understand how cannabis-based information-and specifically what cannabis-based information-should be made available to HCPs so that they are well-equipped to communicate with their patients.

\section{CONCLUSIONS}

The present study provides valuable insight into an expanding area of research. Although cannabis-based research in oncology is growing, information about the role of HCPs 
and their perspectives about the use and prescription of cannabis in cancer care has been lacking. A sizeable gap in professional knowledge and formal training about cannabis among oncology HCPs is evident, although HCPs are willing to become more educated about the topic. A number of clinically-related barriers related to cannabis use and prescription are also faced by HCPs, pointing to the need for further investigation into this topic.

\section{ACKNOWLEDGMENTS}

AM acknowledges the co-authors for their valued insight throughout the project, and the oncology staff members at the Tom Baker Cancer Centre and Holy Cross Cancer Centre for their cooperation and efforts in distributing surveys and informing patients of the study.

This project was funded by the Enbridge Research Chair in Psychosocial Oncology, co-funded by the Canadian Cancer Society Alberta/NWT Division and the Alberta Cancer Foundation (held by LEC) and a Canadian Institutes of Health Research Strategy for Patient-Oriented Research Mentorship Chair in Innovative Clinical Trials (awarded to LEC) and by the Markin Undergraduate Student Research Program in Health and Wellness Studentship (awarded to AM).

\section{CONFLICT OF INTEREST DISCLOSURES}

We have read and understood Current Oncology's policy on disclosing conflicts of interest, and we declare that we have none.

\section{AUTHOR AFFILIATIONS}

*Department of Psychosocial Oncology, Holy Cross Cancer Centre, University of Calgary, and ${ }^{\dagger}$ Department of Oncology, Tom Baker Cancer Centre, University of Calgary, Calgary, AB.

\section{REFERENCES}

1. Maurya N, Velmurugan BK. Therapeutic applications of cannabinoids. Chem Biol Interact 2018;293:77-88.

2. Hill KP. Medical marijuana for treatment of chronic pain and other medical and psychiatric problems: a clinical review. JAMA 2015;313:2474-83.

3. Martell K, Fairchild, Sinha R, et al. Rates of cannabis use in patients with cancer. Curr Oncol 2018;25:219-25.

4. Braun IM, Meyer FL, Gagne JJ, et al. Experts' perspectives on the role of medical marijuana in oncology: a semistructured interview study. Psychooncology 2017;26:1087-92.

5. Abrams DI. Integrating cannabis into clinical cancer care. Curr Oncol 2016;23:S8-14.

6. Reiman A, Welty M, Solomon P. Cannabis as a substitute for opioid-based pain medication: patient self-report. Cannabis Cannabinoid Res 2017;2:160-6.
7. Ware MA, Wang T, Shapiro S, Collet JP on behalf of the COMPASS study team. Cannabis for the management of pain: assessment of safety study (COMPASS). J Pain 2015;16:1233-42.

8. Ware MA, Daeninck P, Maida V. A review of nabilone in the treatment of chemotherapy-induced nausea and vomiting. Ther Clin Risk Manag 2008;4:99-107.

9. Guzmán M. Cannabis for the management of cancer symptoms: THC version 2.0? Cannabis Cannabinoid Res 2018;3:117-19.

10. Braun IM, Wright A, Peteet J, et al. Medical oncologists' beliefs practices and knowledge regarding marijuana used therapeutically: a nationally representative survey study. J Clin Oncol 2018;36:1957-62.

11. Hall W, Degenhardt L. Adverse health effects of non-medical cannabis use. Lancet 2009;374:1383-91.

12. King N, Balneaves LG, Levin GT, et al. Surveys of cancer patients and cancer health care providers regarding complementary therapy use communication and information needs. Integr Cancer Ther 2015;14:515-24.

13. Evanoff AB, Quan T, Dufault C, Awad M, Bierut LJ. Physicians-in-training are not prepared to prescribe medical marijuana. Drug Alcohol Depend 2017;180:151-5.

14. Lee RT, Barbo A, Lopez G, et al. National survey of US oncologists' knowledge, attitudes, and practice patterns regarding herb and supplement use by patients with cancer.JClin Oncol 2014;32:4095-101.

15. Ziemianski D, Capler R, Tekanoff R, Lacasse A, Luconi F, Ware MA. Cannabis in medicine: a national educational needs assessment among Canadian physicians career choice professional education and development. BMC Med Educ 2015;15:1-7.

16. Richardson MA, Mâsse LC, Nanny K, Sanders C. Discrepant views of oncologists and cancer patients on complementary/ alternative medicine. Support Care Cancer 2004;12:797-804.

17. Robinson JH, Callister LC, Associate F, Judith A, Dearing K. Patient-centered care and adherence: definitions and applications to improve outcomes. J Am Acad Nurse Pract 2008;20:600-7.

18. Charalambous A, Radwin L, Berg A, et al. An international study of hospitalized cancer patients' health status nursing care quality perceived individuality in care and trust in nurses: a path analysis. Int J Nurs Stud 2016;61:176-86.

19. Abrams DI. Should oncologists recommend cannabis? Curr Treat Options Oncol 2019;20:59.

20. Hall W. What has research over the past two decades revealed about the adverse health effects of recreational cannabis use? Addiction 2015;110:19-35.

21. Balneaves LG, Alraja A, Ziemianski D, McCuaig F, Ware M. A national needs assessment of Canadian nurse practitioners regarding cannabis for therapeutic purposes. Cannabis Cannabinoid Res 2018;3:66-73. 